\title{
User Testing of a Continuum Manipulator for Assistive Technology
}

\author{
Ryan Coulson ${ }^{1,2}$, Max Kirkpatrick ${ }^{1,3}$, Megan Robinson ${ }^{1,4}$, Meghan Donahue ${ }^{5}$, Devin R. Berg ${ }^{1}$
}

${ }^{1}$ Engineering \& Technology Department, University of Wisconsin-Stout; ${ }^{2}$ Department of Mechanical Engineering, Lafayette College; ${ }^{3}$ Department of Mechanical Engineering, University of South Carolina; ${ }^{4}$ Department of Electrical Engineering and Computer Science, Case Western Reserve University; ${ }^{5}$ Stout Vocational Rehabilitation Institute, University of Wisconsin-Stout

\section{INTRODUCTION}

The use of robots in assistive technology is well-studied, with numerous robotic arms for rehabilitative applications that have been designed and tested to-date, and several that are commercially available [1,2, 3]. These robots are intended to improve independence and quality of life for people who are unable to perform activities of daily living (ADLs) without additional aid. Unfortunately, they are often prohibitively expensive, costing tens of thousands of dollars [4]. Additionally, they pose a risk of harmful collision to their users and must incorporate sophisticated sensors and control methods to ensure the users' safety. This paper evaluates an alternative platform for assistive robotics which alleviates these issues: continuum manipulators.

Continuum manipulators are robots that lack rigid segments and discrete joints [5]. Instead, they function by bending continuously along their length, like the trunk of an elephant or the tentacle of an octopus. To achieve this bending motion, continuum manipulators are often made of soft and flexible materials subject to deformation by external forces. As such, these robots are inherently compliant, meaning they will conform to the shape of their environment. One benefit of their compliance is that continuum manipulators are much safer than traditional rigidlink systems, since they can collide with humans with a reduced chance of physical harm.

Because continuum manipulators are inherently safe, they are well suited for activities that involve coordination close to the human body. For example, grooming activities such as washing and combing hair or bathing would require the robot to touch the person with a disability in areas behind their field of view; miscalculation when positioning a rigid robot could apply uncomfortable pressure to the person. Other activities, such as self-catheterization, are often performed by people with no sensation to their lower extremities. If a rigid robot is unable to sense the lower extremity it is reaching around and stop its motion, it could cause injury, particularly if the user can't feel the robot's pressure. A continuum robot is designed in a way that allows it to react to obstructions without additional sensors and algorithms - if it contacts an extremity, it automatically bends to accommodate it. Because continuum manipulators do not require additional features to ensure the safety of their users, they can also be built with less complexity, and therefore at a lower cost, than traditional assistive robotic arms.

The use of continuum manipulators in assistive technology has been proposed with respect to the ADL of bathing by Ansari et al., 2017 [6], although no user testing of this proposal has been completed. The goal of this research is to demonstrate the viability of continuum manipulators as assistive robots. In this paper, we briefly present the Bendy Assistive Robotic Manipulator (Bendy ARM), a tendon-driven

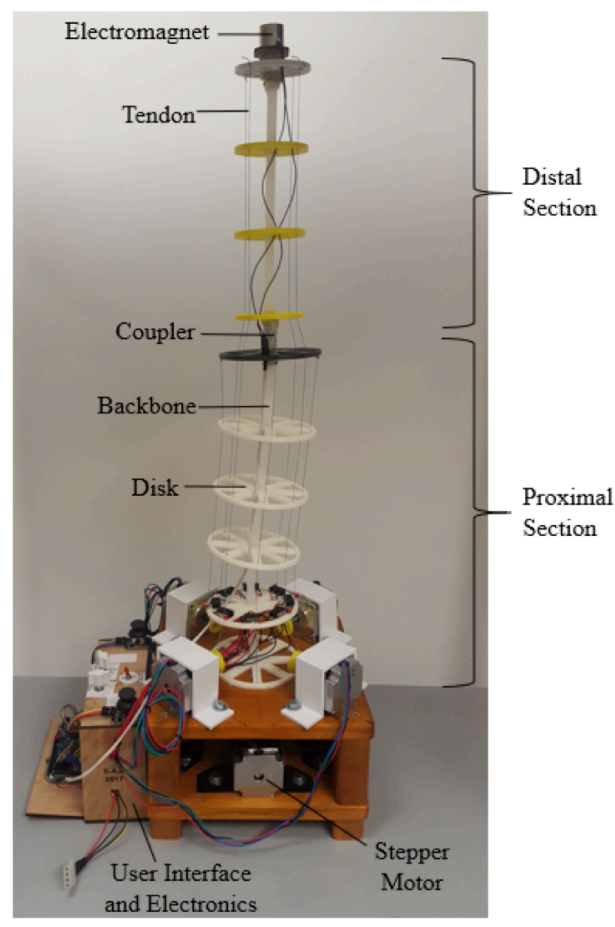

Figure 1. Bendy ARM robot. continuum manipulator for experimentation and user testing.

\section{METHODS}

\section{Design}

Bendy ARM primarily consists of a flexible backbone mounted on a wooden base (see Figure 1). The backbone is divided into two sections. The first (proximal) section, closest to the base, is segmented by six disks. The second (distal) section is segmented by four disks and joined to the proximal section by a metal coupler. Four tendons 
made of 50-pound braided fishing line control each section, terminating at the last disk in the section (the disk furthest from the base of the arm). The four tendons of each section are separated by 90 degrees and driven by four independent stepper motors, which coil and uncoil the tendons around spools. The tendons of the distal section are rotated 45 degrees from the tendons that drive the proximal section.

The result is an eight degree of freedom robot with a maximum reach that is approximated by a sphere of radius equal to the length of the two concatenated sections. The proximal section is $41 \mathrm{~cm}$ long and the distal section is $29 \mathrm{~cm}$ long, yielding a total length, or workspace radius, of $70 \mathrm{~cm}$. However, many of the locations within this sphere are inaccessible due to self-intersection or impractical configuration requirements. Additionally, the two sections of the arm are mechanically coupled - adjusting the tendon lengths in one section affects the position of the other. The mechanical coupling of the robot is a drawback because it makes control less intuitive and control schemes more difficult to implement.

The total cost of the parts used to construct Bendy ARM was below $\$ 550$. Although this robot is only a prototyping platform and many of the components are not suitable for commercial use, the production cost of a commercial model would still be relatively low compared to the assistive robots which are currently available. This price reduction could help increase access to assistive robots and improve quality of life for people with modest incomes living with disabilities.

\section{Control Schemes}

Bendy ARM is teleoperated via a user interface connected directly to the robot. The control panel consists of two joysticks and several switches. The user operates the robot using one of three control schemes: Dual-Joystick, Single-Joystick Segmented, and Single-Joystick Compensative.

In the Dual-Joystick control scheme (henceforth referred to as Dual control), one joystick controls the proximal section and one joystick controls the distal section. When either joystick is pushed in any direction, the corresponding section will bend in the same direction, relative to the position of its base. No attempt at decoupling the two segments is made in this scheme, so adjusting the distal section affects the position of the proximal section, and vice versa. The speed at which the section moves is proportional to displacement of the joystick.

In the Single-Joystick Segmented control scheme (henceforth referred to as Segmented control), only one joystick is used. One advantage of this control scheme is that it allows for users with only one control site to operate the robot. The user can select between three control modes using the 'Mode' switch. In Mode 1, pushing the joystick in any direction will bend both sections simultaneously in that direction such that the whole arm moves as a single unit. This whole-arm movement is useful for gross positioning of the arm. In Mode 2, the joystick controls only the distal section. In Mode 3, the joystick controls only the proximal section. Again, no attempt is made at decoupling the sections.

In the Single-Joystick Compensative control scheme (henceforth referred to as Compensative control), only one joystick is used, with two control modes available via the 'Mode' switch. Mode 1 is the same as in Segmented control, allowing for whole-arm movement. In Mode 2, the joystick controls the distal section, but compensates for the robot's mechanical coupling by moving the proximal section at half speed in the opposite direction. Although this control scheme does not yield perfect compensation, it does enable the distal section to be moved nearly independently of the proximal section. This is useful when attempting fine resolution positioning with the endeffector, since the user can control the distal section without having to consciously compensate for the displacement of the proximal section.

\section{User Testing}

This study seeks insight on which control scheme allows for the most effective and intuitive control of Bendy ARM, as well as how quickly users can learn to effectively operate the robot. To address these questions, two rounds of user testing were designed. Prior to the conduct of any user testing, this project was reviewed by the UW-Stout Institutional Review Board and was determined to be exempt under Category \#1 of the Federal Exempt Guidelines. The specific procedures for each round of user testing are described below. 


\section{Round One}

In Round One of testing, 14 able-bodied users - eight males and six females between 16 and 58 years old - were asked to complete a given task three times, using each control scheme once. The task entailed picking up a metal nut using the electromagnet on the end of the manipulator and dropping it into the cup on the other side of the robot's base.

The experimental procedure began by introducing the three control schemes one at a time, starting with a demonstration, and followed by a one-minute practice period for each control scheme. The order in which the control schemes were introduced and used in trials was systematically varied for each user. After completing the final timed trial, the user was debriefed and given a short survey. The survey asked them to rank the control schemes based on intuitiveness. Additionally, subjects were asked to provide general feedback on their experience, which was taken into consideration when evaluating their results.

\section{Round Two}

The second round of user testing entailed multiple trials of two different tasks to evaluate how user performance improved over time. All three of the subjects involved in Round Two had already participated in Round One. The Compensative control scheme - determined by Round One to be the most effective (discussed further in the following section) - was used for all trials. Testing occurred over multiple sessions, separated by approximately 24 hours. Each session consisted of three trials per task, except for the third session, which consisted of six trials. The specifications of the two tasks (the peg-in-hole task and the drawer task) are detailed below.

The peg-in-hole task was designed to test the user's ability to fine-tune the position of the robot's end-effector. Two pegboards, each with a $2 \times 4$ grid of 5/16" diameter holes, were placed symmetrically on the left and right sides of the robot for this task. The task consisted of using the robot and attached electromagnet to move 1/4" diameter metal bolts from one hole to another. First, the user was required to move a bolt from one hole to a different hole on the same board. Then, they moved a second bolt from a hole on the first board to a hole on the second board. The starting and target holes were varied for every trial such that each trial was different but had the same level of difficulty. Introducing variation was important for ensuring that any measured improvements in task performance were the result of increased proficiency in controlling the robot, rather than memorizing a systematic strategy for accomplishing one invariant task.

The drawer task was more challenging and more closely simulated an activity of daily living (ADL) for which this robot could potentially be used. For this task, the user was asked to open a drawer, remove a metal nut from inside, drop the nut into a cup on the opposite side of the robot, pick up a second nut, place the second nut back in the drawer, and close the drawer. A metal washer was affixed to the plastic drawer so it could be opened using the robot's electromagnet. The position of the drawer was altered with each trial, while holding difficulty constant, to help ensure the validity of the results.

\section{RESULTS AND DISCUSSION}

Table 1 shows the results from Round One of user testing. On average, users were able to complete the given task most quickly using Compensative control, followed by Dual control and then Segmented control. The standard deviations for these data are relatively large, as users demonstrated a wide range of skill levels when using the robot. Examining the average intuition rankings, it can be seen that users rated Compensative control as most intuitive, followed by Dual control and then Segmented control. This result further supports Compensative control as the superior of the three control schemes.
Table 1. Results from Round One of user testing for $\mathbf{n}=$ 14 users. The 'Average Intuition Ranking' column represents an average of all of the intuition rankings provided for each control scheme, where a ranking of ' 1 ' indicated that a scheme was the most intuitive, and a ranking of ' 3 ' indicated that it was the least intuitive.

\begin{tabular}{|l|l|l|l|}
\hline \multicolumn{1}{|c|}{$\begin{array}{c}\text { Control } \\
\text { Scheme }\end{array}$} & $\begin{array}{c}\text { Average } \\
\text { Completion } \\
\text { Time (s) }\end{array}$ & $\begin{array}{c}\text { Standard } \\
\text { Deviation } \\
\text { (s) }\end{array}$ & $\begin{array}{l}\text { Average } \\
\text { Intuition } \\
\text { Ranking }\end{array}$ \\
\hline $\begin{array}{l}\text { Single-Joystick } \\
\text { Compensative }\end{array}$ & 63.00 & 54.61 & 1.86 \\
\hline Dual-Joystick & 72.76 & 42.60 & 1.93 \\
\hline $\begin{array}{l}\text { Single-Joystick } \\
\text { Segmented }\end{array}$ & 96.20 & 55.58 & 2.21 \\
\hline
\end{tabular}

The researchers have proposed an explanation for why Compensative control was the most effective and most intuitive of the three control schemes. Based on observations made during the trials, as well as qualitative feedback from the users, it is suspected that Compensative control imposes the lowest cognitive load of the three 
control schemes, since its second mode automatically compensates for the arm's mechanical coupling, allowing the user to commit more mental energy to other

nuances of the task.

Tables $2 \mathrm{a}$ and $2 \mathrm{~b}$ show results from Round Two of user testing. It can be seen that for both the peg-inhole and drawer tasks, all three users were able to complete the task more quickly, on average, in the last session than the first session. In most cases, these improvements were considerable, with some nearing fifty percent. These results indicate that users are able to substantially increase their proficiency using Bendy ARM with a relatively low amount of practice (the total amount of time spent using the robot between the first and last sessions was less than one hour per user). The significance of this test is that it demonstrates the potential of a continuum manipulator to be effectively used in completing ADLs without requiring a large amount of training.

Future research involving Bendy ARM should include the following: 1) further iterations on the protoype itself, including a method for decoupling the proximal and distal sections, 2) user testing involving subjects from the technology's target population (people with disabilities who have loss of upper limb function), and 3) comparative studies between Bendy ARM and commercially available wheelchair-mounted robotic manipulators (WMRMs), to determine the relative effectiveness of each technology.

\section{CONCLUSION}

Bendy ARM is a tendon-driven continuum manipulator which has the potential to be used as an assistive robot, aiding people with disabilities by increasing their independence in completing ADLs. Continuum manipulators may be better suited for assistive technology than currently commercially available robots due to their inherent safety and relatively low cost. User testing has shown that Bendy ARM can be used to perform multiple tasks (pick-andplace, peg-in-hole, opening and closing drawers) with minimal training. Additionally, this research has yielded quantitative evidence suggesting that users demonstrate significant improvement in using Bendy ARM in a relatively short amount of time. Following further iterations, future research involving Bendy ARM should include user testing with subjects from the target population, as well as statistical comparisons between Bendy ARM and commercially available WMRMs. The findings of this study indicate that there is a strong possibility that continuum manipulators can be applied successfully to the field of assistive technology in the future.

\section{ACKNOWLEDGEMENTS}

This work is supported by the National Science Foundation under Grant No. CNS-1560219. Any opinions, findings, and conclusions or recommendations expressed in this material are those of the authors and do not necessarily reflect the views of the National Science Foundation.

\section{REFERENCES}

[1] Chung, C. S., Wang, H., \& Cooper, R. A. (2013). Functional assessment and performance evaluation for assistive robotic manipulators: Literature review. The Journal of Spinal Cord Medicine, 36(4), 273-289. DOI: 10.1179/2045772313Y.0000000132

[2] Driessen, B. J. F., Evers, H. G., \& v Woerden, J. A. (2001). MANUS-a wheelchair-mounted rehabilitation robot. Proceedings of the Institution of Mechanical Engineers, Part $\mathrm{H}$ : Journal of Engineering in Medicine, 215(3), 285-290. DOI: 10.1243/0954411011535876

[3] Maheu, V., Archambault, P. S., Frappier, J., \& Routhier, F. (2011, June). Evaluation of the JACO robotic arm: Clinico-economic study for powered wheelchair users with upper-extremity disabilities. In Rehabilitation Robotics (ICORR), 2011 IEEE International Conference on (pp. 1-5). IEEE. DOI: 10.1109/ICORR.2011.5975397 
[4] Allin, S., Eckel, E., Markham, H., \& Brewer, B. R. (2010). Recent trends in the development and evaluation of assistive robotic manipulation devices. Physical Medicine and Rehabilitation Clinics of North America, 21(1), 59-77. DOI: 10.1016/j.pmr.2009.09.001

[5] Walker, I. D. (2013). Continuous backbone "continuum" robot manipulators. ISRN Robotics, 2013. DOI: $10.5402 / 2013 / 726506$

[6] Ansari, Y., Manti, M., Falotico, E., Mollard, Y., Cianchetti, M., \& Laschi, C. (2017). Towards the development of a soft manipulator as an assistive robot for personal care of elderly people. International Journal of Advanced Robotic Systems, 14(2), 1729881416687132. DOI: 10.1177/1729881416687132 